\title{
Electroweak Physics, Experimental Aspects
}

\author{
Klaus Mönig \\ DESY-Zeuthen
}

\begin{abstract}
Collider measurements on electroweak physics are summarised. Although the precision on some observables is very high, no deviation from the Standard Model of electroweak interactions is observed. The data allow to set stringent limits on some models for new physics.
\end{abstract}

Plenary Talk at the UK Phenomenology Workshop on Collider Physics, Durham, 1999 


\section{Introduction}

Within the last ten years precision tests of the electroweak theory has been a field of rapid progress. At colliders our knowledge mainly comes from three sources: $\mathrm{e}^{+} \mathrm{e}^{-}$-interactions close to the peak of the Z-resonance, $\mathrm{e}^{+} \mathrm{e}^{-}$-interactions above the $\mathrm{W}$-pair production threshold and $\mathrm{p} \overline{\mathrm{p}}$-interactions above $1 \mathrm{TeV}$ centre of mass energy. In $\mathrm{e}^{+} \mathrm{e}^{-}$close to the Zpeak each of the four LEP experiments has recorded around 4.5 million Z-decays between 1989 and 1995 and their results are final or nearly final. SLD at SLAC has collected around half a million events with polarised electron beams until end of 1998 and first results with the full dataset have been presented. The LEP2 program at energies above $160 \mathrm{GeV}$ is still ongoing. The experiments have mainly analysed their datasets of around $250 \mathrm{pb}^{-1}$ integrated luminosity each at energies up to $189 \mathrm{GeV}$ taken until the end of 1998. The hope is to more than double the luminosity, mainly at energies between 192 and $206 \mathrm{GeV}$. At the TEVATRON the two experiments have collected each $100 \mathrm{pb}^{-1}$ at $\sqrt{s}=1.8 \mathrm{TeV}$ until 1995 . The analysis of this dataset is basically complete. End of next year the TEVATRON will restart for run II to collect a factor 20 or more events.

This report summarises the experimental status of electroweak interactions in the summer 1999. The results in Z-physics, four-fermion physics and two-fermion physics at high energies are presented and the data are interpreted within the framework of the Standard Model.

\section{Precision tests on the $\mathrm{Z}$}

Most results from LEP represent only marginal updates with respect to last year [1]. The most interesting change occurred in the value of the hadronic peak cross section [2]. The experimental data remained constant, however due to additional $\mathcal{O}\left(\alpha^{3}\right)$ corrections for initial state radiation and a new version of the fitting program ZFITTER [3] the value for $\sigma_{0}^{\text {had }}\left(=\frac{12 \pi}{m_{Z}} \frac{\Gamma_{e} \Gamma_{\text {had }}}{\Gamma_{Z}^{2}}\right)$ went up by one standard deviation and due to a decrease in the theoretical error of the luminosity from $0.1 \%$ to $0.06 \%$ its error went down by $30 \%$ so that the that the fitted number of light neutrino species is now two standard deviations below three $\left(N_{\nu}=2.9835 \pm 0.0083\right)$.

SLD have updated their $A_{\mathrm{LR}}$ measurement with the full statistics. The result remains stable, however the error decreases by $10 \%$ [⿴囗⿴囗丨 . In addition SLD presented several updates of the left-right-forward-backward asymmetry for b- and c-quarks measuring the final state coupling parameters $\mathcal{A}_{\mathrm{b}}, \mathcal{A}_{\mathrm{c}}$ [5]. The results are partly already using the full dataset and the error decreased by $30 \%$ with respect to last year.

Figure 1 a) summarises all electroweak results and compares them with the results of the electroweak fit [2]. In general good agreement of the measurements with the Standard Model prediction is found which is reflected also in $\chi^{2} / \mathrm{ndf}=22.9 / 15$ corresponding to a fit probability of $8.6 \%$. The two most discrepant observables are the measurement of $\sin ^{2} \theta_{\text {eff }}^{\ell}$ from $A_{\mathrm{LR}}$ at SLD and the b-forward-backward-asymmetry at LEP which both disagree by roughly two standard deviations from the fit. These two observables are responsible for the $2.7 \sigma$ deviation of $\mathcal{A}_{\mathrm{b}}$ obtained from all relevant LEP and SLD data. It should however be noted that this is about the worst deviation one can construct from the data and that the input variables with the largest weight are statistically dominated.

Figure 1 b) shows the difference in $\chi^{2}$ of the Standard Model fit as a function of the 
Higgs mass for two different values of $\alpha\left(m_{\mathrm{Z}}^{2}\right)$ [6]. Both fits prefer a Higgs mass in the $100 \mathrm{GeV}$ region with a $95 \%$ c.l. upper limit of around $220 \mathrm{GeV}$. The fitted value of $m_{\mathrm{H}}$ is thus consistent with supersymmetric theories as well as with the Standard Model being valid up to the Planck mass.

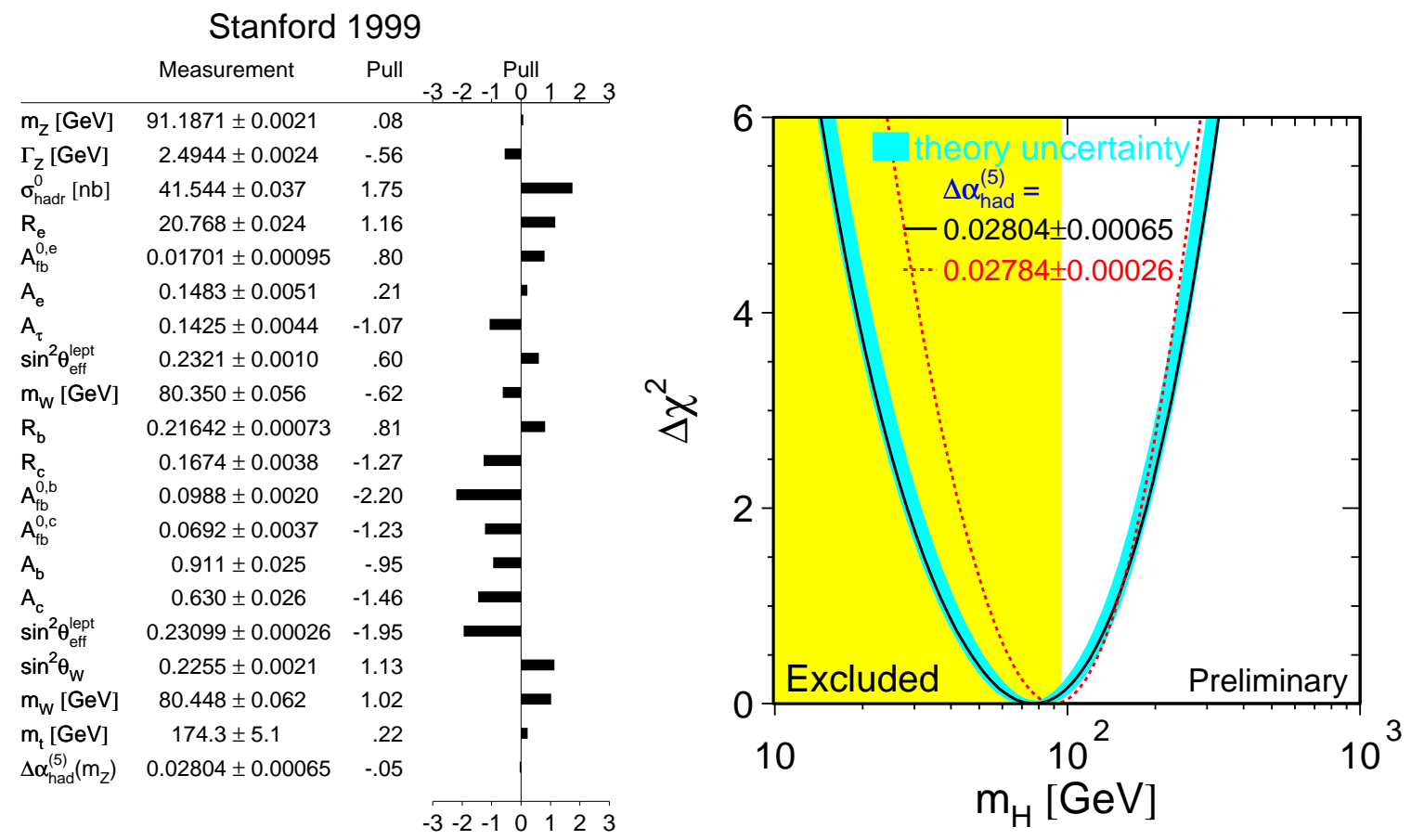

Figure 1: a) comparison of the experimental results with the Standard Model prediction after the electroweak fit. b) $\Delta \chi^{2}$ of the fit as a function of the Higgs-mass

\section{$3 \quad$ W-physics}

The study of W-properties is one of the major issues at LEP2 and at the TEVATRON. At both machines the experiments measure the W-mass and width, its couplings and branching ratios. At LEP the main source of $\mathrm{Ws}$ is $\mathrm{W}$-pair production while at the TEVATRON Ws are mainly produced singly in quark antiquark annihilation.

\subsection{The $\mathrm{W}$-mass and branching ratios}

At LEP the W-mass is determined reconstructing the invariant mass of the final state fermion pairs. For this method the W-pairs with both Ws decaying hadronically [7] and the ones with one $\mathrm{W}$ decaying hadronically and the other into a charged lepton and a neutrino [8] can be used. For the latter the neutrino momentum is identified with the missing momentum in the event. In both cases the resolution is improved by a constrained fit imposing energy and momentum conservation. All experiments also impose directly or indirectly the constraint that the masses of the two pairs are (about) equal. The constrained fits improve the experimental resolution significantly and make the mass, reconstructed from the fully hadronic decays, practically independent of the energy scale 
of the experiment. On the other hand they make the results dependent on the knowledge of the beam energy and on initial state radiation. For the fully hadronic decays the analysis is complicated by final state effects [9]. The $\mathrm{W}$ flight distance is about $0.1 \mathrm{fm}$ which a factor 10 smaller than the typical fragmentation radius. The quarks and gluons from the different Ws can therefor interact with each other during fragmentation. Recent studies on this effect give mass shifts of the order of $50 \mathrm{MeV}$ which is taken as a systematic error. In addition Bose Einstein correlations between the final state pions have to be taken into account. It is theoretically unclear if these correlations should be visible for particles from different $\mathrm{Ws}$ and the experimental situation is contradictory. From model studies an error of about $20 \mathrm{MeV}$ is taken as systematic error.

At $p \bar{p}$ colliders the $W$ mass can be measured only for leptonic $W$ decays [10]. Since an unknown longitudinal momentum is lost in the beampipe only the transverse momentum of the neutrino can be reconstructed. The $\mathrm{W}$-mass is thus reconstructed from the transverse mass distribution of the charged lepton/neutrino pair. The main limitations at present are the understanding of the energy scale of the detector and of the hadronic recoil. Both are calibrated with leptonic $\mathrm{Z}$ decays, so that the corresponding errors are mainly of statistical nature. The remaining theoretical errors are small at present, but could become relevant again at run II.

Figure 2 summarises the $\mathrm{W}$-masses obtained with the different methods [8, 7, 10]. It can be seen that the results agree very well and that the precision of the direct measurement starts to match the one of the prediction from the accurate $\mathrm{Z}$ data.

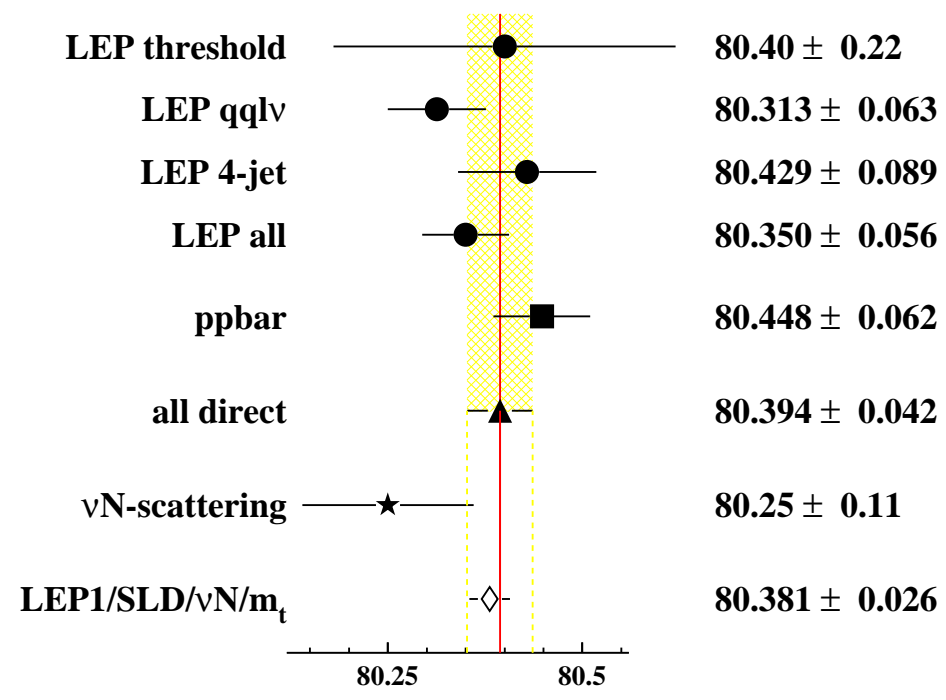

Figure 2: Measurements of $m_{\mathrm{W}}$ using the different methods.

From the W-mass spectra at LEP or from the high end of the transverse mass spectrum at the TEVATRON also the $\mathrm{W}$ width can be determined. However to date only CDF has done the measurement at the TEVATRON and only L3 has included the $189 \mathrm{GeV}$ data. Both measurements (LEP: $\Gamma_{\mathrm{W}}=2.12 \pm 0.20 \mathrm{GeV}$ [11], CDF: $\Gamma_{\mathrm{W}}=2.055 \pm 0.125 \mathrm{GeV}$ [10]) are in agreement with the SM prediction of $2.08 \mathrm{GeV}$.

At LEP the $\mathrm{W}$ branching ratios are measured together with the $\mathrm{W}$-pair cross section. Under the assumption that all $\mathrm{W}$ decays are visible, each $\mathrm{W}$ pair event can be classified according to the two decays and the branching ratios can be measured simul- 
taneously with the cross section. At the TEVATRON the experiments measure the ratio $R=\frac{\sigma(\mathrm{p} \overline{\mathrm{p}} \rightarrow \mathrm{W} \rightarrow \ell \nu)}{\sigma\left(\mathrm{p} \rightarrow \mathrm{\textrm {p }} \rightarrow \ell^{+} \ell^{-}\right)}$. The ratio of production cross sections $\frac{\sigma(\mathrm{p} \overline{\mathrm{p}} \rightarrow \mathrm{W})}{\sigma(\mathrm{p} \overline{\mathrm{p}} \rightarrow \mathrm{Z})}$ is then imposed from theory and $\operatorname{Br}(\mathrm{Z} \rightarrow \ell \ell)$ is taken from LEP, so that $\operatorname{Br}(\mathrm{W} \rightarrow \ell \nu)$ can be obtained. The partial width $\Gamma(\mathrm{W} \rightarrow \ell \nu)$ is well known from theory, so that the branching ratio $\operatorname{Br}(\mathrm{W} \rightarrow \ell \nu)$ measurements can be converted into $\Gamma_{\mathrm{W}}$ measurements. All direct and indirect $\Gamma_{\mathrm{W}}$ measurements are compared with the SM prediction in fig. 3. The indirect measurement from the TEVATRON is slightly high $(1.7 \sigma)$ [12]. However the precision of the direct measurements is currently much too bad to confirm or disprove the disagreement. The LEP number [13] is in between the SM and the TEVATRON result. However, $\operatorname{Br}(\mathrm{W} \rightarrow \ell \nu)$ directly is not sensitive to invisible $\mathrm{W}$ decays which should show up in a too small $\mathrm{W}$-pair or a too large single $\mathrm{W}$ cross section.

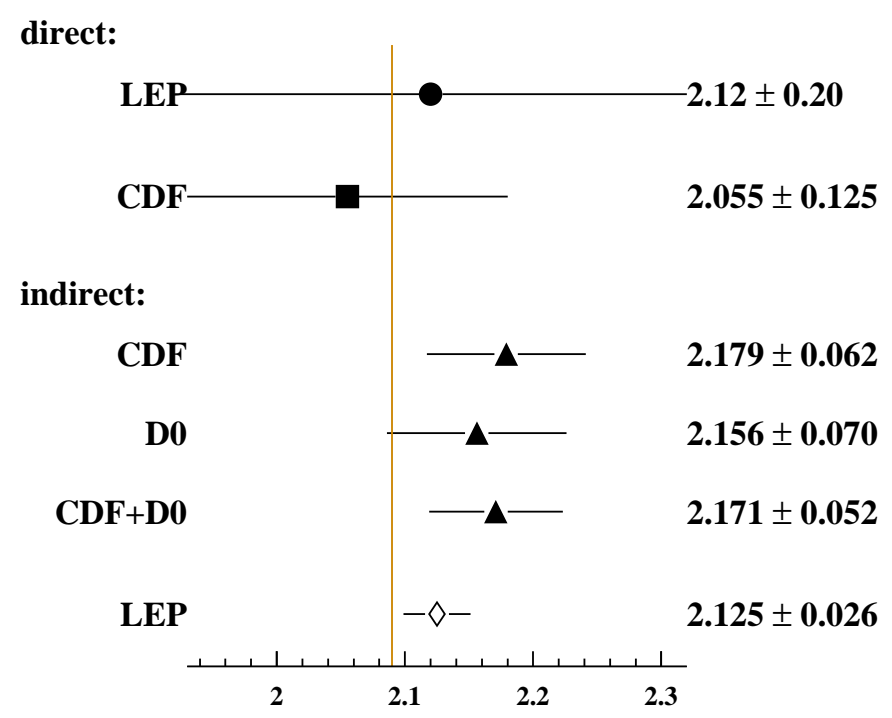

Figure 3: Direct and indirect measurements of $\Gamma_{\mathrm{W}}$.

\subsection{Measurement of gauge boson couplings}

The most sensitive channel for the measurement of triple gauge boson couplings is Wpair production at LEP which contains, apart from a $\nu_{e}$ t-channel exchange graph also graphs with s-channel $\gamma$ or Z-exchange. However in this channel, contributions from anomalous ZWW and $\gamma \mathrm{WW}$ graphs cannot be separated. At the TEVATRON the most sensitive channel is $\mathrm{W} \gamma$ pair production, which is sensitive to $\gamma \mathrm{WW}$ couplings only. Some information is also obtained from WZ and WW pairs. Also at LEP the $\gamma \mathrm{WW}$ couplings can be separated using single $\mathrm{W}$ production, which is dominated by $\gamma \mathrm{W}$ fusion and single $\gamma$ production. The experiments usually parametrise the triple gauge couplings with the five parameters $g_{1}^{Z}, \kappa_{\gamma}, \kappa_{Z}, \lambda_{\gamma}, \lambda_{Z}$ defined in [14]. The $\gamma \mathrm{WW}$ and ZWW couplings are then related by $\Delta \kappa_{\gamma}=-\frac{\cos ^{2} \theta_{W}}{\sin ^{2} \theta_{W}}\left(\Delta \kappa_{Z}-\Delta g_{1}^{Z}\right)$ and $\lambda_{\gamma}=\lambda_{Z}$. Table 1 summarises the single parameter fits for the different channels [15, 16, 17]. Again, no deviations from the predictions are observed. The LEP experiments have also performed two parameter fits for any pair of coupling-parameters. Some of the parameters are significantly correlated, and also in these fits all results agree with the Standard Model. 


\begin{tabular}{|l|c|c|c|}
\hline & $\Delta g_{1}^{Z}$ & $\Delta \kappa_{\gamma}$ & $\lambda_{\gamma}$ \\
\hline LEP all & $0.04 \pm 0.08$ & $-0.01 \pm 0.03$ & $-0.04 \pm 0.04$ \\
LEP single W & & $-0.09 \pm 0.12$ & \\
D0 & & $-0.08 \pm 0.34$ & $0.00 \pm 0.09$ \\
\hline
\end{tabular}

Table 1: 1-parameter fit results for anomalous triple gauge couplings

\section{2-fermion physics at high energies}

2-fermion production at high energies, away from the Z-resonance peak, can be used to test a variety of new physics effects. At LEP one has access to cross sections for lepton- and quark-pair production. For quarks the total hadronic cross section and the fraction of b- and c-quarks within the hadronic sample can be measured. In addition one can measure the forward-backward asymmetry for leptons, b- and c-quarks. At energies above the $\mathrm{Z}$, in a large part of the events a high energy photon is radiated from the initial state, so that the effective centre of mass energy of the $\mathrm{e}^{+} \mathrm{e}^{-}$-interaction $\left(\sqrt{s^{\prime}}\right)$ is close to the Z-mass. However, it is no problem to reject these events with high efficiency using the energy and acolinearity of the measured particles or jets. As an example figure 1 shows the comparison of the cross sections measured at LEP with the Standard Model prediction. At the TEVATRON a similar process is available with Drell-Yan production of lepton pairs, however no new results have been presented in the recent past. All 2-fermion results agree well with the SM prediction[18]. So they can be used to set limits on a variety

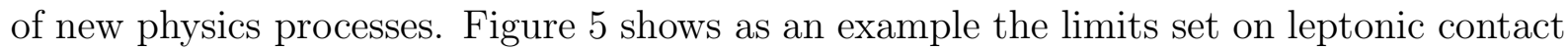
interaction for different helicity structures [19]. As another example figure 6 shows the limits on a possible Z' in different models obtained by OPAL [20]. The tight limits on Z-Z' mixing come from the LEP1 precision data while the mass limit for zero mixing is from LEP2. For no mixing the TEVATRON sets limits of comparable size from Drell-Yan production.

\section{Conclusions}

The analysis of the Z-precision data from LEP and SLD is basically finished. Despite the enormous precision of the data no deviation from the Standard Model has been found. The data favour a light Higgs boson which is equally predicted in supersymmetric theories and in the Standard Model if it is required to be valid up to the Planck scale. At higher energies at LEP and at the TEVATRON the $\mathrm{W}$-mass has been measured with comparable precision to the Z-data, leading to the same conclusions. In addition a variety of other observables have been measured. Since all agree with the prediction of the electroweak theory stringent limits on new physics can be set. 


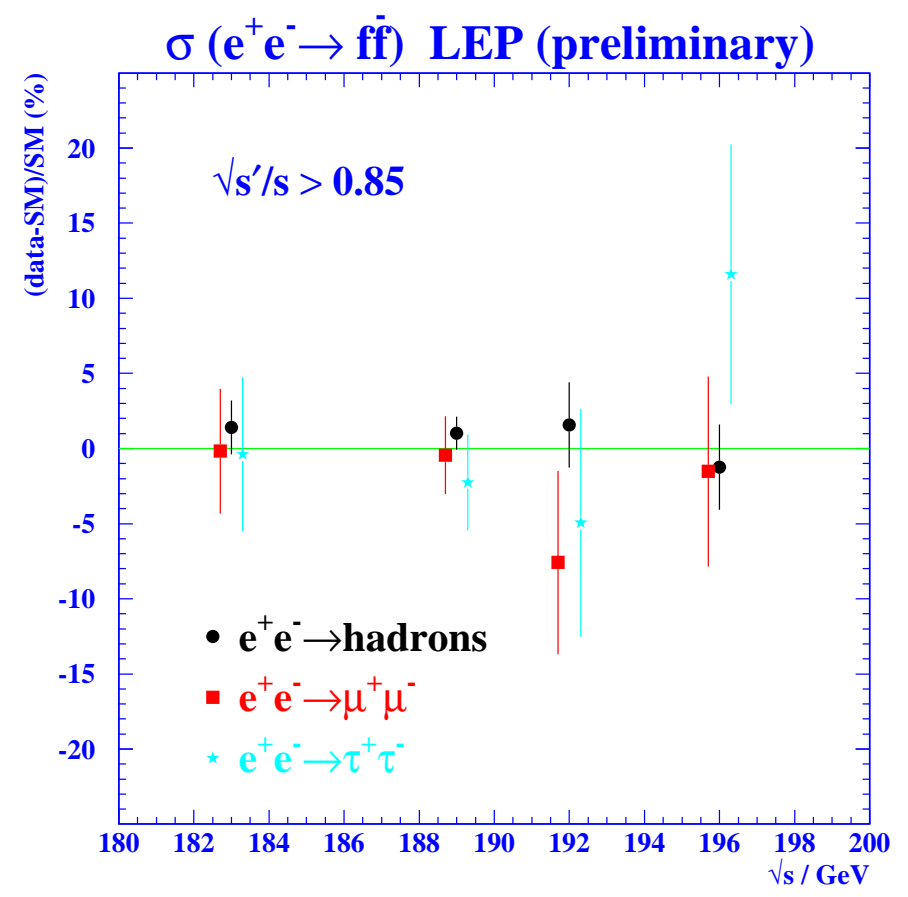

Figure 4: Comparison of the 2-fermion production cross section at LEP2 with the SM prediction.

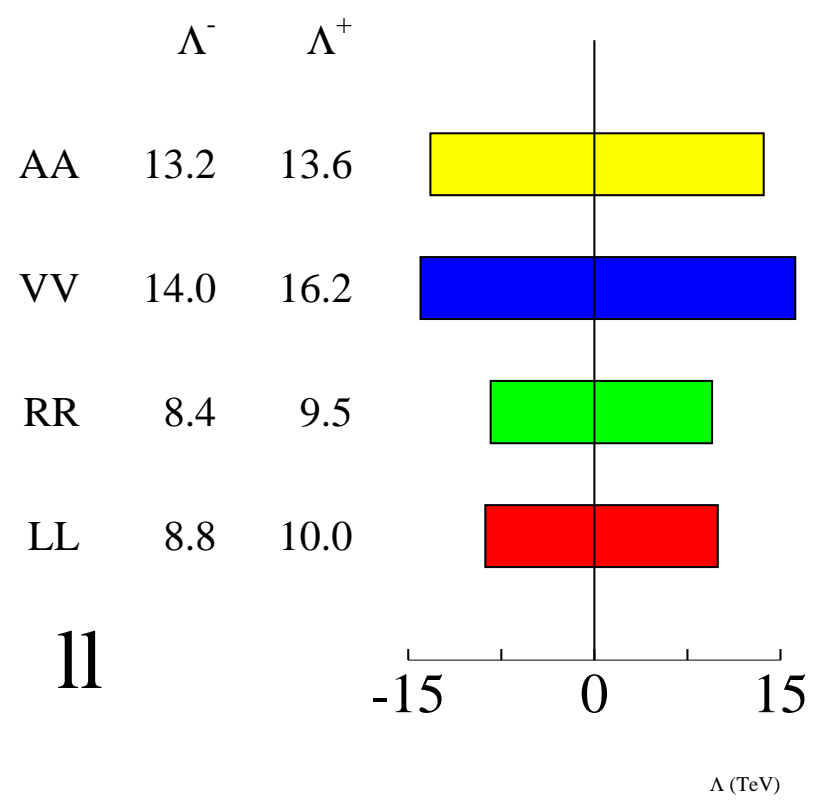

Figure 5: Contact interaction limits (95\%c.l.) at LEP2. 


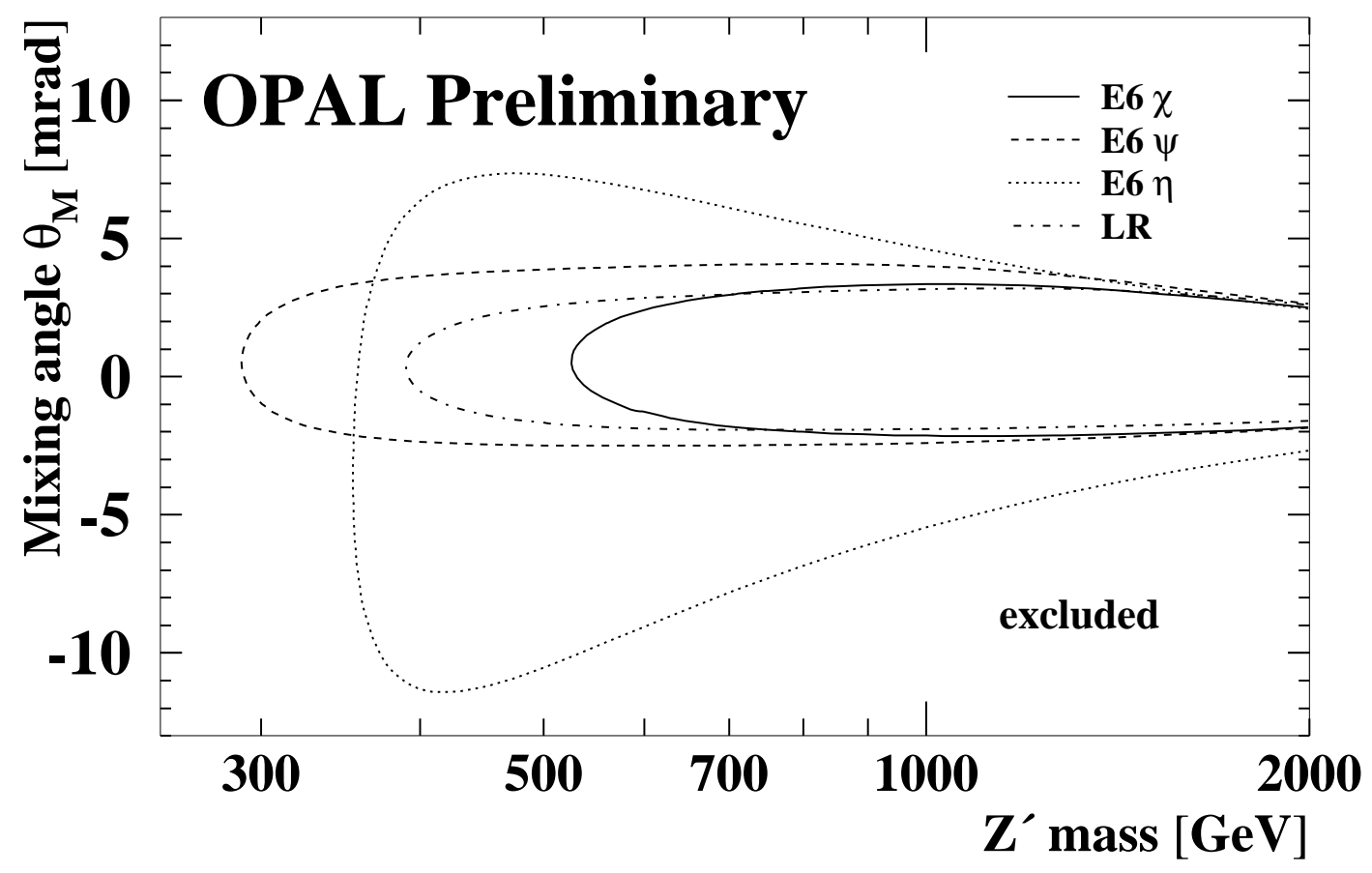

Figure 6: Z' limits (95\%c.l.) for various models from OPAL. 


\section{Acknowledgements}

It is a pleasure to thank the organisers of the workshop for the nice and friendly atmosphere at the conference and the conveners and participants of the electroweak working group for the useful discussions during the meeting. Especially I would like to thank Mark Lancaster and Niels Kjaer for their advice preparing the talk and for reading the manuscript.

\section{References}

[1] The LEP collaborations, A Combination of Preliminary Electroweak Measurements and Constraints on the Standard Model, CERN-EP/99-15.

[2] G. Quast, EW review at LEP1, talk given at EPS 99 Tampere, to appear in the proceedings.

[3] D. Bardin et al., Z. Phys. C44 (1989) 493; Comp. Phys. Comm. 59 (1990) 303; Nucl. Phys. B351(1991) 1; Phys. Lett. B255 (1991) 290 and CERN-TH 6443/92 (May 1992).

[4] J. Brau, Electroweak Precision Measurements with Leptons, talk given at EPS 99 Tampere, to appear in the proceedings.

[5] S. Fahey, Measurements of Quark Coupling Asymmetries at the $Z^{0}$, talk given at EPS 99 Tampere, to appear in the proceedings.

[6] Z. Kunszt, these proceedings

[7] R. Chierici, $W$ mass from fully leptonic and mixed decays at LEP, talk given at EPS 99 Tampere, to appear in the proceedings.

[8] L. Mir, W mass from fully hadronic decays at LEP, talk given at EPS 99 Tampere, to appear in the proceedings.

[9] J. Schiek, New Results on Fragmentation Effects Associated with W Production, talk given at EPS 99 Tampere, to appear in the proceedings.

[10] B. Carithers, Measurement of the W Mass at the Tevatron, talk given at EPS 99 Tampere, to appear in the proceedings.

[11] D. Charlton, Precision EW at LEPII, talk given at LP 99 Stanford, to appear in the proceedings.

[12] CDF Electroweak Group, W,Z Cross Sections + W Width Measurement http://www-cdf.fnal.gov/physics/ewk/xsec_width_new.html

[13] A .Barczyk, W properties at LEP, talk given at EPS 99 Tampere, to appear in the proceedings.

[14] K. Gaemers, G. Gounaris, Zeit. Phys. C1 (1979), 259;

K. Hagiwara et al., Nucl. Phys. B282 (1987), 253. 
[15] A. Macchiolo, Anomalous couplings from $\mathrm{e}^{+} \mathrm{e}^{-} \rightarrow W^{+} W^{-}$, talk given at EPS 99 Tampere, to appear in the proceedings.

[16] O. Iouchtchenko, Other anomalous WWV couplings (mainly single $W$ production), talk given at EPS 99 Tampere, to appear in the proceedings.

[17] J. Ellison, $W$ and $Z$ properties at the Tevatron, talk given at EPS 99 Tampere, to appear in the proceedings.

[18] M. Minard, $\mathrm{e}^{+} \mathrm{e}^{-} \rightarrow \mathrm{f} \bar{f}$ at LEPQ, talk given at EPS 99 Tampere, to appear in the proceedings.

[19] H. Rick, Tests of the validity of the $S M$ through $\mathrm{e}^{+} \mathrm{e}^{-} \rightarrow \mathrm{f} \overline{\mathrm{f}}, \mathrm{e}^{+} \mathrm{e}^{-} \rightarrow \gamma s$, talk given at EPS 99 Tampere, to appear in the proceedings.

[20] OPAL Collaboration, Limits on a Z' Boson from $\mathrm{e}^{+} \mathrm{e}^{-}$to fermion pair Cross-sections and Asymmetries OPAL PN-372, contributed paper to EPS 99 Tampere HEP'99 19 . 